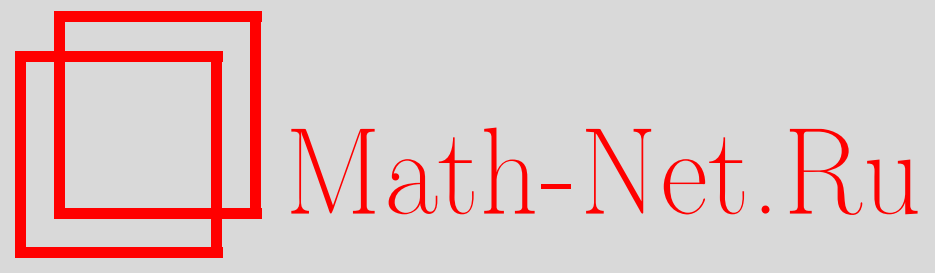

P. К. Тагиев, В. М. Габибов, Об одной задаче оптимального управления для уравнения теплопроводности с интегральным граничным условием, Вестн. Сам. гос. техн. ун-та. Сер. Физ.-мат. науки, 2016, номер 1, 54-64

DOI: https://doi.org/10.14498/vsgtu1463

Использование Общероссийского математического портала MathNet.Ru подразумевает, что вы прочитали и согласны с пользовательским соглашением

http: //www.mathnet.ru/rus/agreement

Параметры загрузки:

IP: 18.234 .197 .8

26 апреля 2023 г., $11: 58: 43$

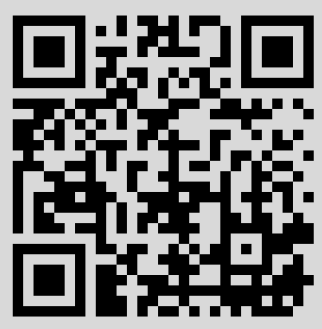


УДК 517.977

\title{
ОБ ОДНОЙ ЗАДАЧЕ ОПТИМАЛЬНОГО УПРАВЛЕНИЯ ДЛЯ УРАВНЕНИЯ ТЕПЛОПРОВОДНОСТИ С ИНТЕГРАЛЬНЫМ ГРАНИЧНЫМ УСЛОВИЕМ
}

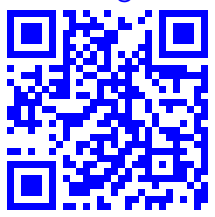

\author{
P. К. Тагиев ${ }^{1}$, В. М. Габибов ${ }^{2}$ \\ 1 Бакинский государственный университет, \\ Азербайджан, AZ-1148, Баку, ул. 3. Халилова, 23. \\ 2 Ленкоранский государственный университет, \\ Азербайджан, AZ-4200, Ленкорань, пр-т Ази Асланова, 50.
}

\section{Аннотация}

В данной работе рассматривается задача оптимального управления для уравнения теплопроводности с интегральным граничным условием. Управляющими функциями являются коэффициент и свободный член уравнения состояния, а также свободный член интегрального граничного условия. Коэффициент и свободный член уравнения состояния являются элементами пространства Лебега, а свободный член интегрального условия - элементом пространств Соболева. Функционал цели является финальным. Исследованы вопросы корректности постановки задачи оптимального управления в слабой топологии пространства управлений. Доказано, что в рассматриваемой задаче существует хотя бы одно оптимальное управление, множество оптимальных управлений слабо компактно в пространстве управлений, а любая минимизирующая последовательность управлений функционала цели слабо сходится к множеству оптимальных управлений. Доказана дифференцируемость по Фреше функционала цели на множестве допустимых управлений. Получены формулы для дифференциала градиента функционала цели. Установлено необходимое условие оптимальности в форме вариационного неравенства.

Ключевые слова: оптимальное управление, уравнения теплопроводности, необходимое условие оптимальности.

Введение. Задачи оптимального управления процессами, описываемыми уравнениями с частными производными параболического типа, встречаются в различных приложениях $[1,2]$. Такие задачи наиболее полно исследованы в случаях, когда краевые условия для уравнений состояний являются классическими, т.е. локальными [1-6 и др.]. Однако известны многочисленные задачи физики, техники, биологии и др., в которых процессы описываются уравнениями параболического типа, где краевые условия являются неклассическими,

(C) 2016 Самарский государственный технический университет.

\section{Образец для цитирования}

Т а г и е в Р. К., Габ и бо в В. М. Об одной задаче оптимального управления для уравнения теплопроводности с интегральным граничным условием // Вестн. Сам. гос. техн. ун-та. Сер. Физ.-мат. науки, 2016. Т. 20, № 1. С. 54-64. doi: 10.14498/vsgtu1463.

\section{Сведения об авторах}

Рафик Каландар огль Тагиев (д.ф.-м.н., проф.; r.tagiyev@list.ru; автор, ответственный за переписку), профессор, каф. оптимизации и управления.

Вахаб Мехти Габибов (vahab.hebibov@mail.ru), старший преподаватель, каф. физики, математики и информатики. 
или нелокальными [7-9]. Среди нелокальных краевых задач для уравнений параболического типа особое место занимают краевые задачи с интегральными граничными условиями [10-13]. Задачи оптимального управления для уравнений параболического типа с нелокальными краевыми условиями, в том числе с интегральными граничными условиями, изучены мало.

В данной работе рассматривается задача оптимального управления для уравнения теплопроводности с интегральным граничным условием. Исследованы вопросы корректности постановки задачи в слабой топологии пространства управлений, доказана дифференцируемость по Фреше функционала цели, получены формулы для его дифференциала и градиента, установлено необходимое условие оптимальности в форме вариационного неравенства.

1. Постановка задачи. Пусть управляемый процесс описывается в

$$
Q_{T}=\{(x, t): 0<x<l, 0<t<T\}
$$

следующей нелокальной начально-краевой задачей для уравнения теплопроводности с интегральным граничным условием:

$$
\begin{gathered}
u_{t}-u_{x x}+v_{1}(x, t) u=v_{2}(x, t), \quad(x, t) \in Q_{T}, \\
u(x, 0)=\varphi(x), \quad 0 \leqslant x \leqslant l, \\
u_{x}(0, t)=0, \quad u_{x}(l, t)=\int_{0}^{l} H(x) u_{x}(x, t) d x+v_{3}(t), \quad 0<t \leqslant T,
\end{gathered}
$$

где $\varphi(x) \in W_{2}^{1}(0, l), H(x) \in \stackrel{\circ}{W_{2}^{1}}(0, l)$ - заданные функции; $u=u(x, t)=$ $=u(x, t ; v)$ - функция состояния задачи $(1)-(3)$, соответствующая управлению $v=\left(v_{1}(x, t), v_{2}(x, t), v_{3}(t)\right)$.

Введем множество допустимых управлений

$$
\begin{aligned}
& V=\left\{v=\left(v_{1}(x, t), v_{2}(x, t), v_{3}(t)\right) \in H=L_{2}\left(Q_{T}\right) \times L_{2}\left(Q_{T}\right) \times W_{2}^{1}(0, T):\right. \\
& \left.\left|v_{1}(x, t)\right| \leqslant d_{1} \quad \text { п.в. на } \quad Q_{T},\left\|v_{2}\right\|_{L_{2}\left(Q_{T}\right)} \leqslant d_{2}, \quad\left\|v_{3}\right\|_{W_{2}^{1}(0, T)} \leqslant d_{3}\right\} \text {, }
\end{aligned}
$$

где $d_{1}, d_{2}, d_{3}>0$ - заданные числа.

Поставим следующую задачу оптимального управления: минимизировать функционал

$$
J(v)=\int_{0}^{l}|u(x, T ; v)-y(x)|^{2} d x
$$

на решениях $u=u(x, t ; v)$ краевой задачи $(1)-(3)$, соответствующих всем допустимым управлениям $v \in V$. Здесь $y(x) \in W_{2}^{1}(0, l)$ - заданная функция. Эту задачу ниже будем называть задачей (1)-(5).

Обозначения используемых в работе функциональных пространств соответствуют принятым в [14, с. 24]. Ниже положительные постоянные, не зависящие от оцениваемых величин и допустимых управлений, обозначаются через $M$.

Под решением краевой задачи (1)-(3), соответствующим управлению $v \in V$, будем понимать обобщенное решение из $V_{2}^{1,0}\left(Q_{T}\right)$, т.е. функцию $u=u(x, t)=$ $=u(x, t ; v)$ из $V_{2}^{1,0}\left(Q_{T}\right)$, удовлетворяющую интегральному тождеству 


$$
\begin{aligned}
\int_{Q_{T}}\left(-u \eta_{t}\right. & \left.+u_{x} \eta_{x}+v_{1}(x, t) u \eta\right) d x d t=\int_{Q_{T}} v_{2}(x, t) \eta d x d t+ \\
& +\int_{0}^{l} \varphi(x) \eta(x, 0) d x+\int_{0}^{T}\left(\int_{0}^{l} H(x) u_{x}(x, t) d x+v_{3}(t)\right) \eta(l, t) d t
\end{aligned}
$$

при любой функции $\eta=\eta(x, t) \in W_{2}^{1}\left(Q_{T}\right)$, равной нулю при $t=T$.

Используя методики работ [13], [14, с. 165-171], можно показать, что при сделанных предположениях краевая задача (1)-(3) имеет единственное обобщенное решение из $V_{2}^{1,0}\left(Q_{T}\right)$ при каждом фиксированном $v \in V$ и справедлива априорная оценка

$$
\begin{aligned}
& \|u\|_{V_{2}^{1,0}\left(Q_{T}\right)} \equiv \max _{0 \leqslant t \leqslant T}\|u(x, t ; v)\|_{L_{2}(0, l)}+\left\|u_{x}\right\|_{L_{2}\left(Q_{T}\right)} \leqslant \\
& \leqslant M\left(\left\|v_{2}\right\|_{L_{2}\left(Q_{T}\right)}+\|\varphi\|_{L_{2}(0, l)}+\left\|v_{3}\right\|_{L_{2}(0, T)}\right) .
\end{aligned}
$$

Более того, обобщенное решение из $V_{2}^{1,0}\left(Q_{T}\right)$ краевой задачи (1)-(3) также принадлежит пространству $W_{2}^{2,1}\left(Q_{T}\right)$ и для него справедлива оценка

$$
\|u\|_{W_{2}^{2,1}\left(Q_{T}\right)} \leqslant M\left(\left\|v_{2}\right\|_{L_{2}\left(Q_{T}\right)}+\|\varphi\|_{W_{2}^{1}(0, l)}+\left\|v_{3}\right\|_{W_{2}^{1}(0, T)}\right) .
$$

Из оценки (7) следует, что функционал (5) определен на $V$ и принимает конечные значения.

2. Корректность постановки задачи. Корректность постановки задачи (1)(5) в слабой топологии пространства $H=L_{2}\left(Q_{T}\right) \times L_{2}\left(Q_{T}\right) \times W_{2}^{1}(0, T)$ устанавливает следующая теорема.

Теорема 1. Пусть выполнены условия, принятые при постановке задачи (1)-(5). Тогда множество оптимальных управлений задачи (1)-(5)

$$
V_{*}=\left\{v_{*} \in V: J\left(v_{*}\right)=J_{*} \equiv \inf \{J(v): v \in V\}\right\}
$$

непусто, $V_{*}$ слабо компактно в $H$ и любал минимизирующая последовательность $\left\{v^{(n)}\right\}$ функиионала (5) слабо в $H$ сходится $к$ множеству $V_{*}$.

Доказательство. Покажем, что функционал (5) непрерывен на $V$ в слабой топологии пространства $H$.

Пусть $\left\{v^{(n)}\right\}=\left\{\left(v_{1}^{(n)}(x, t), v_{2}^{(n)}(x, t), v_{3}^{(n)}(t)\right)\right\} \subset V-$ произвольная последовательность такая, что $\left\{v^{(n)}\right\} \rightarrow v=\left(v_{1}(x, t), v_{2}(x, t), v_{3}(t)\right)$ слабо в $H$ к некоторому элементу $v=\left(v_{1}(x, t), v_{2}(x, t), v_{3}(t)\right) \in H$ :

$$
\begin{gathered}
v_{i}^{(n)}(x, t) \rightarrow v_{i}(x, t) \text { слабо в } L_{2}\left(Q_{T}\right), \quad i=1,2, \\
v_{3}^{(n)}(t) \rightarrow v_{3}(t) \text { слабо в } W_{2}^{1}(0, T) .
\end{gathered}
$$

Так как $V \subset H$ - выпуклое замкнутое в $H$ множество, оно слабо замкнуто и в гильбертовом пространстве $H$, поэтому $v=\left(v_{1}(x, t), v_{2}(x, t), v_{3}(t)\right) \in V$.

Из компактности вложения $W_{2}^{1}(0, T) \rightarrow C[0, T][14$, с. 84] и (10) следует, что

$$
v_{3}^{(n)}(t) \rightarrow v_{3}(t) \text { сильно в } C[0, T] .
$$


Кроме этого, в силу однозначной разрешимости задачи (1)-(3) каждому управлению $v^{(n)} \in V$ соответствует единственное решение $u^{(n)}=u\left(x, t ; v^{(n)}\right) \in$ $W_{2}^{2,1}\left(Q_{T}\right)$ задачи $(1)-(3)$ при $v=v^{(n)}$ и справедлива оценка

$$
\left\|u^{(n)}\right\|_{W_{2}^{2,1}\left(Q_{T}\right)} \leqslant M, \quad n=1,2, \ldots,
$$

т.е. последовательность $\left\{u^{(n)}\right\}$ равномерно ограничена в норме пространства $W_{2}^{2,1}\left(Q_{T}\right)$.

Известно [15, с. 33, 39], что пространство $W_{2}^{2,1}\left(Q_{T}\right)$ компактно вложено в $L_{r}\left(Q_{T}\right)$ для любого конечного $r \geqslant 2$. Кроме того, следы элементов $u(x, t) \in$ $W_{2}^{2,1}\left(Q_{T}\right)$ определены при каждом фиксированном $t \in[0, T]$ как элементы $W_{2}^{1}(0, l)$ и справедлива оценка [16, с. 98]

$$
\sup _{0 \leqslant t \leqslant T}\|u(x, t)\|_{W_{2}^{1}(0, l)} \leqslant M\|u\|_{W_{2}^{2,1}\left(Q_{T}\right)} \cdot
$$

Отсюда и из компактности вложения $W_{2}^{1}(0, l) \rightarrow C[0, l][14$, с. 84] следует, что отображение $\left.u \rightarrow u\right|_{t=T}$ пространства $W_{2}^{2,1}\left(Q_{T}\right)$ в $C[0, l]$ компактно. Тогда из (12) следует, что из последовательности $\left\{u^{(n)}\right\}$ можно извлечь подпоследовательность $\left\{u^{\left(n_{k}\right)}\right\}$ такую, что

$$
\begin{gathered}
u^{\left(n_{k}\right)}(x, t) \rightarrow u(x, t) \text { слабо в } W_{2}^{2,1}\left(Q_{T}\right), \\
u^{\left(n_{k}\right)}(x, t) \rightarrow u(x, t) \text { сильно в } L_{2}\left(Q_{T}\right), \\
u^{\left(n_{k}\right)}(x, T) \rightarrow u(x, T) \text { сильно в } C[0, l],
\end{gathered}
$$

где $u=u(x, t)$ - некоторый элемент из $W_{2}^{2,1}\left(Q_{T}\right)$. Далее на основе соотношений (6), (9), (11), (12), (14)-(16) и ограничений на входные данные и управление $v$ можно показать, что $u=u(x, t)$ - решение задачи $(1)-(3)$, соответствующее управлению $v$, т.е. $u(x, t)=u(x, t ; v)$. Таким образом, установлено, что при выполнении соотношений (9), (10) справедлива сходимость

$$
u^{\left(n_{k}\right)}(x, T)=u\left(x, T ; v^{\left(n_{k}\right)}\right) \rightarrow u(x, T)=u(x, T ; v) \text { сильно в } C[0, l] .
$$

Используя единственность решения краевой задачи (1)-(3), соответствующее каждому фиксированному управлению $v \in V$, можно показать, что соотношение (17) справедливо не только для подпоследовательности $\left\{u^{\left(n_{k}\right)}\right\}$, но и для всей последовательности $\left\{u^{(n)}\right\}$ :

$$
u^{(n)}(x, T)=u\left(x, T ; v^{(n)}\right) \rightarrow u(x, T)=u(x, T ; v) \text { сильно в } C[0, l] .
$$

Тогда, используя соотношение $(18)$ из $(5)$, получаем, что $J\left(v^{(n)}\right) \rightarrow J(v)$ при $n \rightarrow \infty$. Таким образом, установлено, что функционал (5) слабо в $H$ непрерывен на множестве $V$. Тогда, применяя результат из [1, с. 49], получаем, что задача (1)-(5) корректно поставлена в слабой топологии пространства $H$, т.е. справедливы все утверждения теоремы 1. 
3. Дифференцируемость функционала цели и необходимое условие оптимальности. Для задачи (1)-(5) введем сопряженное состояние $\psi=\psi(x, t)=$ $=\psi(x, t ; v)$ как решение задачи

$$
\begin{gathered}
\psi_{t}+\psi_{x x}-v_{1}(x, t) \psi-H^{\prime}(x) \psi(l, t)=0, \quad(x, t) \in Q_{T}, \\
\psi(x, T)=2[u(x, T ; v)-y(x)], \quad 0 \leqslant x \leqslant l \\
\psi_{x}(0, t)=0, \quad \psi_{x}(l, t)=0, \quad 0 \leqslant t<T .
\end{gathered}
$$

Под решением краевой задачи (19)-(21), соответствующим управлению $v \in V$, будем понимать обобщенное решение из $V_{2}^{1,0}\left(Q_{T}\right)$, т.е. функцию $\psi=$ $=\psi(x, t)=\psi(x, t ; v)$ из $V_{2}^{1,0}\left(Q_{T}\right)$, удовлетворяющую интегральному тождеству

$$
\begin{aligned}
\int_{Q_{T}}\left(\psi \eta_{t}+\psi_{x} \eta_{x}+v_{1}(x, t) \psi \eta\right) d x d t & -\int_{0}^{T}\left(\int_{0}^{l} H(x) \eta_{x}(x, t) d x\right) \psi(l, t) d t= \\
& =2 \int_{0}^{T}[u(x, T ; v)-y(x)] \eta(x, T) d x
\end{aligned}
$$

при любой функции $\eta=\eta(x, t) \in W_{2}^{1}\left(Q_{T}\right)$, равной нулю при $t=0$.

Используя методики работ [13], [14, с. 165-171], можно показать, что для каждого заданного $v \in V$ краевая задача (19)-(21) имеет единственное обобщенное решение из $V_{2}^{1,0}\left(Q_{T}\right)$. Более того, это решение принадлежит пространству $W_{2}^{2,1}\left(Q_{T}\right)$ и справедлива оценка

$$
\|\psi\|_{W_{2}^{2,1}\left(Q_{T}\right)} \leqslant M\|u(x, T ; v)-y(x)\|_{W_{2}^{1}(0, l)} .
$$

Учитывая здесь неравенство (13) и оценки (8), получаем оценку

$$
\|\psi\|_{W_{2}^{2,1}\left(Q_{T}\right)} \leqslant M\left[\left\|v_{2}\right\|_{L_{2}\left(Q_{T}\right)}+\|\varphi\|_{W_{2}^{1}(0, l)}+\|y\|_{W_{2}^{1}(0, l)}+\left\|v_{3}\right\|_{W_{2}^{1}(0, T)}\right] .
$$

Теорема 2. Пусть выполнены условия теоремы 1. Тогда функиионал (5) дифберениируем по Фреше на $V$ и его дифберенииал в точке $v \in V$ при приращении $\Delta v=\left(\Delta v_{1}, \Delta v_{2}, \Delta v_{3}\right) \in H$ определяется равенством

$$
d J(v, \Delta v)=\int_{Q_{T}}\left(-u \psi \Delta v_{1}+\psi \Delta v_{2}\right) d x d t+\int_{0}^{T} \psi(l, t ; v) \Delta v_{3}(t) d t
$$

До каз а тель ст в о. Пусть $v, v+\Delta v \in V$ - произвольные управления и

$$
\Delta u=\Delta u(x, t)=u(x, t ; v+\Delta v)-u(x, t ; v), \quad u=u(x, t)=u(x, t ; v) .
$$

Из условий (1)-(3) следует, что $\Delta u$ является решением из $W_{2}^{2,1}\left(Q_{T}\right)$ задачи

$$
\begin{gathered}
\Delta u_{t}-\Delta u_{x x}+\left(v_{1}+\Delta v_{1}\right) \Delta u=-\Delta v_{1} u+\Delta v_{2}, \quad(x, t) \in Q_{T}, \\
\Delta u(x, 0)=0, \quad 0 \leqslant x \leqslant l \\
\Delta u_{x}(0, t)=0, \quad \Delta u_{x}(l, t)=\int_{0}^{l} H(x) \Delta u_{x}(x, t) d x+\Delta v_{3}(t), \quad 0<t \leqslant T .
\end{gathered}
$$


Можно показать, что для решения задачи (26)-(28) верна оценка [14, c. 165-173], [16, с. 197-209]

$$
\|\Delta u\|_{W_{2}^{2,1}\left(Q_{T}\right)} \leqslant M\left[\left\|\Delta v_{1} u\right\|_{L_{2}\left(Q_{T}\right)}+\left\|\Delta v_{2}\right\|_{L_{2}\left(Q_{T}\right)}+\left\|\Delta v_{3}\right\|_{W_{2}^{1}(0, T)}\right] .
$$

Известно [15, с. 33, 39], что вложение $W_{2}^{2,1}\left(Q_{T}\right) \rightarrow L_{\infty}\left(Q_{T}\right)$ ограничено. Используя этот факт, имеем

$$
\left\|\Delta v_{1} u\right\|_{L_{2}\left(Q_{T}\right)} \leqslant\left\|\Delta v_{1}\right\|_{L_{2}\left(Q_{T}\right)}\|u\|_{L_{\infty}\left(Q_{T}\right)} \leqslant M\left\|\Delta v_{1}\right\|_{L_{2}\left(Q_{T}\right)}\|u\|_{W_{2}^{2,1}\left(Q_{T}\right)} .
$$

Из последней оценки и из оценок (29), (8) получаем

$$
\|\Delta u\|_{W_{2}^{2,1}\left(Q_{T}\right)} \leqslant M\|\Delta v\|_{H}
$$

Приращение функционала (5) имеет вид

$$
\begin{aligned}
\Delta J(v)=J(v+ & \Delta v)-J(v)= \\
& =2 \int_{0}^{l}[u(x, T ; v)-y(x)] \Delta u(x, T) d x+\int_{0}^{l}|\Delta u(x, T)|^{2} d x .
\end{aligned}
$$

$\mathrm{C}$ помощью решения $\psi=\psi(x, t)=\psi(x, t ; v)$ краевой задачи $(19)-(21)$ преобразуем приращение (31). Решение краевой задачи (26)-(28) удовлетворяет равенству

$$
\begin{aligned}
\int_{Q_{T}}\left(\Delta u_{t} \psi\right. & \left.+\Delta u_{x} \psi_{x}+v_{1} \Delta u \psi\right) d x d t-\int_{0}^{T}\left(\int_{0}^{l} H(x) \Delta u_{x}(x, t) d x\right) \psi(l, t) d t= \\
=\int_{Q_{T}}\left(-u \psi \Delta v_{1}+\psi \Delta v_{2}\right) d x d t+\int_{0}^{T} \psi(l, t ; v) \Delta v_{3}(t) d t- & -\int_{Q_{T}} \psi \Delta u \Delta v_{1} d x d t
\end{aligned}
$$

Если в тождестве (22) положим $\eta=\Delta u$ и полученное равенство вычтем из (32), то придем к равенству

$$
\begin{aligned}
& 2 \int_{0}^{l}[u(x, T ; v)-y(x)] \Delta u(x, T) d x= \\
& \quad=\int_{Q_{T}}\left(-u \psi \Delta v_{1}+\psi \Delta v_{2}\right) d x d t+\int_{0}^{T} \psi(l, t ; v) \Delta v_{3}(t) d t-\int_{Q_{T}} \psi \Delta u \Delta v_{1} d x d t .
\end{aligned}
$$

Учитывая последнее равенство, (31) запишем в виде

$$
\Delta J(v)=\int_{Q_{T}}\left(-u \psi \Delta v_{1}+\psi \Delta v_{2}\right) d x d t+\int_{0}^{T} \psi(l, t ; v) \Delta v_{3}(t) d t+R,
$$

где

$$
R=\int_{0}^{l}|\Delta u(x, T)|^{2} d x-\int_{Q_{T}} \psi \Delta u \Delta v_{1} d x d t
$$


Покажем, что сумма первых двух слагаемых в правой части равенства (33), т.е. выражение (25), при заданном $v \in V$ определяет линейный ограниченный функционал от $\Delta v$ в $H$. Линейность функционала (25) по $\Delta v$ очевидна. Используя ограниченность вложения $W_{2}^{2,1}\left(Q_{T}\right) \rightarrow L_{\infty}\left(Q_{T}\right)[15$, с. 33, 39], оценку (13) для функции $\psi$, неравенство Коши-Буняковского и оценки (8), (23), получаем неравенства

$$
\begin{gathered}
|d J(v, \Delta v)|=\left|\int_{Q_{T}}\left(-u \psi \Delta v_{1}+\psi \Delta v_{2}\right) d x d t+\int_{0}^{T} \psi(l, t ; v) \Delta v_{3}(t) d t\right| \leqslant \\
\leqslant\|u\|_{L_{\infty}\left(Q_{T}\right)}\|\psi\|_{L_{2}\left(Q_{T}\right)}\left\|\Delta v_{1}\right\|_{L_{2}\left(Q_{T}\right)}+\|\psi\|_{L_{2}\left(Q_{T}\right)}\left\|\Delta v_{2}\right\|_{L_{2}\left(Q_{T}\right)}+ \\
+\|\psi(l, t ; v)\|_{L_{2}(0, T)}\left\|\Delta v_{3}\right\|_{L_{2}(0, T)} \leqslant \\
\leqslant M\|u\|_{W_{2}^{2,1}\left(Q_{T}\right)}\|\psi\|_{L_{2}\left(Q_{T}\right)}\left\|\Delta v_{1}\right\|_{L_{2}\left(Q_{T}\right)}+\|\psi\|_{L_{2}\left(Q_{T}\right)}\left\|\Delta v_{2}\right\|_{L_{2}\left(Q_{T}\right)}+ \\
+M\|\psi\|_{W_{2}^{2,1}\left(Q_{T}\right)}\left\|\Delta v_{3}\right\|_{L_{2}(0, T)} \leqslant M\|\Delta v\|_{H},
\end{gathered}
$$

из которых следует ограниченность функционала (25).

Кроме этого, используя ограниченность вложения $W_{2}^{2,1}\left(Q_{T}\right) \rightarrow L_{\infty}\left(Q_{T}\right)$, неравенство (13) для функции $\Delta u$ и оценки (24), (30), для остаточного члена $R$, определяемого равенством (34), получаем оценку

$$
\begin{gathered}
|R|=\left|\|\Delta u(x, T)\|_{L_{2}(0, l)}^{2}-\int_{Q_{T}} \psi \Delta u \Delta v_{1} d x d t\right| \leqslant \\
\leqslant M\|\Delta u\|_{W_{2}^{2,1}\left(Q_{T}\right)}^{2}+\|\psi\|_{L_{\infty}\left(Q_{T}\right)}\|\Delta u\|_{L_{2}\left(Q_{T}\right)}\left\|\Delta v_{1}\right\|_{L_{2}\left(Q_{T}\right)} \leqslant \\
\leqslant M\|\Delta v\|_{H}^{2}+M\|\psi\|_{W_{2}^{2,1}\left(Q_{T}\right)}\|\Delta v\|_{H}\left\|\Delta v_{1}\right\|_{L_{2}\left(Q_{T}\right)} \leqslant M\|\Delta v\|_{H}^{2} .
\end{gathered}
$$

Учитывая в (33) эту оценку, заключаем, что функционал (5) дифференцируем по Фреше на $V$ и его дифференциал определяется выражением (25).

Теперь получим явную формулу для градиента функционала (5). Поставим следующую краевую задачу для определения функции $\theta=\theta(t)=\theta(t ; v)$ :

$$
\begin{gathered}
-\theta^{\prime \prime}(t)+\theta(t)=\psi(l, t ; v), \quad 0<t<T, \\
\theta^{\prime}(0)=\theta^{\prime}(T)=0 .
\end{gathered}
$$

Под решением задачи (35), (36) при заданном $v \in V$ будем понимать функцию $\theta=\theta(t)=\theta(t ; v)$ из $W_{2}^{1}(0, T)$, удовлетворяющую интегральному тождеству

$$
\int_{0}^{T}\left[\theta^{\prime}(t) \eta^{\prime}(t)+\theta(t) \eta(t)\right] d t=\int_{0}^{T} \psi(l, t ; v) \eta(t) d t
$$

при любой функции $\eta=\eta(t) \in W_{2}^{1}(0, T)$.

Можно показать [14, с. 112-114], что краевая задача $(35),(36)$ при заданном $v \in V$ однозначно разрешима в $W_{2}^{1}(0, T)$.

ТеОрема 3. Пусть выполнены условия теоремы 1. Тогда градиент функиионала (5) в произвольной точке $v \in V$ определяется равенством

$$
J^{\prime}(v)=(-u(x, t ; v) \psi(x, t ; v), \psi(x, t ; v), \theta(t ; v))
$$


$u$ отображение $v \rightarrow J^{\prime}(v)$ непреръвно действует из $V$ в $H$.

Доказа тельств о. Пусть $v, v+\Delta v \in V$ - произвольные управления, где $\Delta v=\left(\Delta v_{1}, \Delta v_{2}, \Delta v_{3}\right) \in H$ - приращение управления на элементе $v=$ $=\left(v_{1}, v_{2}, v_{3}\right) \in V$.

Полагая в тождестве $(37) \eta=\Delta v_{3}$, получаем равенство

$$
\int_{0}^{T}\left[\theta^{\prime}(t) \Delta v_{3}^{\prime}(t)+\theta(t) \Delta v_{3}(t)\right] d t=\int_{0}^{T} \psi(l, t ; v) \Delta v_{3}(t) d t
$$

учитывая которое в (25), имеем

$$
\begin{aligned}
d J(v, \Delta v)=\int_{Q_{T}}\left(-u \psi \Delta v_{1}\right. & \left.+\psi \Delta v_{2}\right) d x d t+ \\
& +\int_{0}^{T}\left[\theta^{\prime}(t) \Delta v_{3}^{\prime}(t)+\theta(t) \Delta v_{3}\right] d t=\left\langle J^{\prime}(v), \Delta v\right\rangle_{H} .
\end{aligned}
$$

Отсюда следует, что градиент функционала (5) определяется равенством (38). Доказательство непрерывности отображения $v \rightarrow J^{\prime}(v)$ проводится, как в работе [5], и ввиду ограниченности объема работы оно здесь опускается.

СледствиЕ. Пусть выполнены условия теоремы 1. Тогда для оптимальности управления $v_{*}=\left(v_{1 *}, v_{2 *}, v_{3 *}\right) \in V$ в задаче $(1)-(5)$ необходимо, чтобъ неравенство

$$
\begin{aligned}
\int_{Q_{T}}\left[-u_{*} \psi_{*}\left(v_{1}-v_{1 *}\right)+\psi_{*}\left(v_{2}-\right.\right. & \left.\left.v_{2 *}\right)\right] d x d t+ \\
& +\int_{0}^{T}\left[\theta_{*}^{\prime}\left(v_{3}^{\prime}-v_{3 *}^{\prime}\right)+\theta_{*}\left(v_{3}-v_{3 *}\right)\right] d t \geqslant 0
\end{aligned}
$$

выполнялосъ для любого $v=\left(v_{1}, v_{2}, v_{3}\right) \in V$, где $u_{*}=u\left(x, t ; v_{*}\right), \psi_{*}=\psi\left(x, t ; v_{*}\right)$, $\theta_{*}=\theta\left(t ; v_{*}\right)-$ решения задач $(1)-(3) ;(19)-(21) ;(35)$, (36) соответственно npu $v=v_{*}$.

Действительно, множество $V$, определяемое равенством (4), выпукло в $H$. Кроме этого, согласно формулировкам теорем 2 и 3, функционал (5) непрерывно дифференцируем по Фреше на $V$. Тогда в силу теоремы 5 из [1, с. 28] на элементе $v_{*} \in V_{*}$ необходимо выполнение неравенства

$$
\left\langle J^{\prime}(v), v-v_{*}\right\rangle_{H} \geqslant 0
$$

при всех $v \in V$. Отсюда и из (38) следует справедливость неравенства (39).

\section{ORCIDs}

Рафик Каландар оглы Тагиев: http://orcid.org/0000-0002-4185-4219

Вахаб Мехти Габибов: http://orcid.org/0000-0003-2610-2008

\section{БИБЛИОГРАФИЧЕСКИЙ СПИСОК}

1. Васильев Ф. П. Методы решения экстремальных задач. Задачи минимизации в функциональных пространствах, регуляризация, аппроксимачия. М.: Наука, 1981. 400 с. 
2. Егоров А. И. Оптимальное управление тепловыми и диффузионными прочессами. М.: Наука, 1978. 436 с.

3. Лионс Ж.-Л. Оптимальное управление системами, описываемыми уравнениями с частными производными. М.: Мир, 1972. 416 с.

4. Искендеров А. Д., Тагиев Р. К. Задачи оптимизации с управлениями в коэффициентах параболического уравнения // Дифферени. уравнения, 1983. Т. 19, № 8. С. 1324-1334.

5. Тагиев Р. К. Оптимальное управление коэффициентами в параболических системах // Дифферени. уравнения, 2009. Т. 45, № 10. С. 1492-1501.

6. Тагиев Р. К. Задача оптимального управления для квазилинейного параболического уравнения с управлениями в коэффициентах и с фазовыми ограничениями // Дифберени. уравнения, 2013. Т. 49, №3. С. 380-392. doi: 10.1134/S0374064113030138.

7. Ионкин Н. И. Решение одной краевой задачи теории теплопроводности с неклассическим краевым условием // Дифферени. уравнения, 1977. Т. 13, № 2. С. 294-304.

8. Самарский А. А. О некоторых проблемах теории дифференциальных уравнений // Дифферени. уравнения, 1980. Т. 16, № 11. С. 1925-1935.

9. Нахушев А. М. Уравнения математической биологии. М.: Высш. шк., 1995. 301 с.

10. Иванчов Н. И. Краевые задачи для параболического уравнения с интегральными условиями // Дифферени. уравнения, 2004. Т. 40, № 4. С. 547-564.

11. Кожанов А. И. О разрешимости краевой задачи с нелокальным граничным условием для линейных параболических уравнений // Вестн. Сам. гос. техн. ун-та. Сер. Физ.мат. науки, 2004. №30. С. 63-69. doi: 10.14498/vsgtu308.

12. Пулькина Л. С. Нелокальная задача для уравнения теплопроводности / Неклассические уравнения математической физики: Международный семинар, посвященный 60летию со дня рождения профессора В. Н. Врагова (Новосибирск, 3-5 октября 2005 г.); ред. А. И. Кожанов. Новосибирск: Инс-т мат. СО РАН, 2005. С. 231-239.

13. Данилкина О. Ю. Об одной нелокальной задаче для уравнения теплопроводности с интегральным условием // Вестн. Сам. гос. техн. ун-та. Сер. Физ.-мат. науки, 2007. № 1(14). C. 5-9. doi : 10.14498/vsgtu480.

14. Ладыженская О. А. Краевые задачи математической физики. М.: Наука, 1973. 408 с.

15. Лионс Ж.-Л. Управление сингулярными распределенными системами. М.: Мир, 1987. $368 \mathrm{c.}$

16. Ладыженская О. А., Солонников В. А., Уральцева Н. Н. Линейные и квазилинейные уравнения параболического типа. М.: Наука, 1967. 736 с.

Поступила в редакцию 22/XI/2015;

в окончательном варианте - 22/I/2016;

принята в печать $-26 / \mathrm{II} / 2016$. 
Vestn. Samar. Gos. Techn. Un-ta. Ser. Fiz.-mat. nauki

[J. Samara State Tech. Univ., Ser. Phys. \& Math. Sci.], 2016, vol. 20, no. 1, pp. 54-64

ISSN: 2310-7081 (online), 1991-8615 (print)

doi: http://dx.doi.org/10.14498/vsgtu1463

MSC: 49J20, 35K20

\title{
ON OPTIMAL CONTROL PROBLEM FOR THE HEAT EQUATION WITH INTEGRAL BOUNDARY CONDITION
}

\author{
R. K. Tagiev ${ }^{1}$, V. M. Habibov ${ }^{2}$ \\ 1 Baku State University, \\ 23, Z. Khalilov st., Baku, AZ-1148, Azerbaijan. \\ 2 Lankaran State University, \\ 50, Azi Aslanov prospectus, Lankaran, AZ-4200, Azerbaijan.
}

\begin{abstract}
In this paper we consider the optimal control problem for the heat equation with an integral boundary condition. Control functions are the free term and the coefficient of the equation of state and the free term of the integral boundary condition. The coefficients and the constant term of the equation of state are elements of a Lebesgue space and the free term of the integral condition is an element of Sobolev space. The functional goal is the final. The questions of correct setting of optimal control problem in the weak topology of controls space are studied. We prove that in this problem there exist at least one optimal control. The set of optimal controls is weakly compact in the space of controls and any minimizing sequence of controls of a functional of goal converges weakly to the set of optimal controls. There is proved Frechet differentiability of the functional of purpose on the set of admissible controls. The formulas for the differential of the gradient of the purpose functional are obtained. The necessary optimality condition is established in the form of variational inequality.
\end{abstract}

Keywords: optimal control, heat equation, necessary optimality condition.

\section{ORCIDs}

Rafiq K. Tagiev: http://orcid.org/0000-0002-4185-4219

Vahab M. Habibov: http://orcid.org/0000-0003-2610-2008

\section{REFERENCES}

1. Vasil'ev F. P. Metody resheniia ekstremal'nykh zadach. Zadachi minimizatsii v funktsional'nykh prostranstvakh, reguliarizatsiia, approksimatsiia [Methods for solving extremal

(C) 2016 Samara State Technical University.

Please cite this article in press as:

T a g i e v R. K., H a bi bov V. M. On optimal control problem for the heat equation with integral boundary condition, Vestn. Samar. Gos. Tekhn. Univ., Ser. Fiz.-Mat. Nauki [J. Samara State Tech. Univ., Ser. Phys. \& Math. Sci.], 2016, vol. 20, no. 1, pp. 54-64. doi: 10.14498/vsgtu1463. (In Russian)

\section{Authors Details:}

Rafiq K. Tagiev (Dr. Phys. \& Math. Sci.; r.tagiyev@list.ru; Corresponding Author), Professor, Dept. of Optimization and Control.

Vahab M. Habibov (vahab.hebibov@mail.ru), Senior Lecturer, Dept. of Physics, Mathematics and Computer Science. 
problems. Minimization problems in function spaces, regularization, approximation]. Moscow, Nauka, 1981, 400 pp. (In Russian)

2. Egorov A. I. Optimal'noe upravlenie teplovymi i diffuzionnymi protsessami [Optimal control of thermal and diffusion processes]. M., Nauka, 1978, 436 pp. (In Russian)

3. Lions J. L. Optimal control of systems governed by partial differential equations, Die Grundlehren der mathematischen Wissenschaften, 170. Berlin, Heidelberg, New York, Springer-Verlag, 1971, xi+396 pp.

4. Iskenderov A. D., Tagiev R. K. Problems of optimization with controls in the coefficients of a parabolic equation, Differ. Equ., 1983, vol. 19, no. 8, pp. 990-999.

5. Tagiev R. K. Optimal coefficient control in parabolic systems, Differ. Equ., 2009, vol. 45, no. 10, pp. 1526-1535. doi: 10.1134/S0012266109100164.

6. Tagiev R. K. Optimal control problem for a quasilinear parabolic equation with controls in the coefficients and with state constraints, Differ. Equ., 2013, vol.49, no. 3, pp. 369-381. doi : 10.1134/S0012266113030129.

7. Ionkin N. I. Solution of a boundary-value problem in heat conduction with a nonclassical boundary condition, Differ. Equ., 1977, vol. 13, no. 2, pp. 204-211.

8. Samarskii A. A. On some problems of the theory of differential equations, Differ. Uravn., 1980, vol. 16, no. 11, pp. 1925-1935 (In Russian).

9. Nakhushev A. M. Uravneniia matematicheskoi biologii [Equations of mathematical biology]. Moscow, Vyssh. shk., 1995, 301 pp. (In Russian)

10. Ivanchov N. I. Boundary value problems for a parabolic equation with integral conditions, Differ. Equ., 2004, vol. 40, no. 4, pp. 591-609. doi: 10.1023/B:DIEQ.0000035796.56467.44.

11. Kozhanov A. I. On the solvability of a boundary-value problem with a nonlocal boundary condition for linear parabolic equations, Vestn. Samar. Gos. Tekhn. Univ. Ser. Fiz.-Mat. Nauki [J. Samara State Tech. Univ., Ser. Phys. \& Math. Sci.], 30, 2004, pp. 63-69 (In Russian). doi: 10.14498/vsgtu308.

12. Pul'kina L. S. Nonlocal problem for the heat equation, Nonclassical equations of mathematical physics, Proceedings of the seminar dedicated to the 60th birthday of Prof. V. N. Vragov (October 3-5, 2005, Novosibirsk); ed. A. I. Kozhanov. Novosibirsk, 2005, pp. 231-239 (In Russian).

13. Danilkina O. Yu. On one nonlocal problem for the heat equation with an integral condition, Vestn. Samar. Gos. Tekhn. Univ. Ser. Fiz.-Mat. Nauki [J. Samara State Tech. Univ., Ser. Phys. \& Math. Sci.], 2007, no. 1(14), pp. 5-9 (In Russian). doi: 10.14498/vsgtu480.

14. Ladyzhenskaia O. A. Kraevye zadachi matematicheskoi fiziki [Boundary-value problems of mathematical physics]. Moscow, Nauka, 1973, 408 pp. (In Russian)

15. Lions J. L. Control of Distributed Singular Systems. Paris, Gauthier-Villars, 1985, 552 pp.

16. Ladyzhenskaia O. A., Solonnikov V. A., Ural'tseva N. N. Lineinye i kvazilineinye uravneniia parabolicheskogo tipa [Linear and quasilinear equations of parabolic type]. Moscow, Nauka, 1967, 736 pp. (In Russian)

Received 22/XI/2015; received in revised form $22 / \mathrm{I} / 2016$; accepted 26/II/2016. 